\title{
Population density predicts outcome from out-of-hospital cardiac arrest in Victoria, Australia
}

\section{Ziad Nehme BEmergHlth(Hons) Research Coordinator ${ }^{1}$ and PhD Candidate ${ }^{2}$ \\ Emily Andrew BBiomedSc Manager Research Governancel \\ Peter A Cameron MBBS, MD Adjunct Professor and Research ${ }^{2}$ \\ Janet E Bray $\mathrm{RN}, \mathrm{PhD}$ Research Fellow2 \\ Stephen A Bernard MBBS, MD Adjunct Professor, ${ }^{2}$ and Senior Intensive Care Physician $^{3}$ \\ Ian T Meredith BSc(Hons), MBBS, PhD Professor of Cardiology, ${ }^{4}$ and Director of Cardiology ${ }^{5}$ \\ Karen Smith BSc(Hons), PhD Associate Professor, 6 and Manager Research and Evaluation?}

1 Ambulance Victoria Melbourne, VIC.

2 Department of Epidemiology and Preventive Medicine

Monash University, Melbourne, VIC.

3 The Alfred Hospital, Melbourne, VIC

4 Department of Medicine Monash University,

Melbourne, VIC.

5 Monash Medical Centre, Melbourne, VIC

6 Discipline of Emergency Medicine, University of Western Australia, Perth

ziad.nehme@ ambulance.vic.gov.au

MJA 2014; 200: 471-475 doi: 10.5694/mjal3.10856
O ut-of-hospital cardiac arrest (OHCA) remains a significant national and global health problem, and is estimated to affect almost 25000 Australians annually. ${ }^{1}$ Timely access to emergency medical service (EMS) treatment remains an integral aspect of the chain of survival, and has an important role in determining the provision and performance of EMSs across both urban and regional communities. ${ }^{2}$ Regional variation in the characteristics and outcomes of OHCA has been described internationally, although few authors have examined the contribution of population density on their reported outcomes.

Previous reports from Australia have found that population factors contribute to the variation in outcomes observed for conditions such as stroke, ${ }^{3}$ acute myocardial infarction $^{4}$ and major trauma. ${ }^{5}$ Similarly, OHCA outcomes have been examined for spatial relationships across areas of Europe, 6,7 North America ${ }^{8,9}$ and Japan; 10 however, methods and findings have been inconsistent. In Australia, several authors have shown poorer survival outcomes for OHCA patients in regional areas, although these reports failed to account for the potential effect of population density on survival. ${ }^{11,12}$

In this study, we examine the contribution of population density to the reported incidence, characteristics and outcome of OHCA in Victoria, Australia.

\section{Methods}

\section{Study design}

We retrospectively extracted data from the Victorian Ambulance Cardiac Arrest Registry (VACAR) for all nonEMS witnessed OHCA events between 1 January 2003 and 31 December 2011. Adult patients aged more than 17 years who suffered a cardiac arrest of presumed cardiac aetiology were included in the sample. Ethics approval for this

\section{Abstract}

Objective: To examine the impact of population density on incidence and outcome of out-of-hospital cardiac arrest (OHCA).

Design, setting and participants: Data were extracted from the Victorian Ambulance Cardiac Arrest Registry for all adult OHCA cases of presumed cardiac aetiology attended by the emergency medical service (EMS) between 1 January 2003 and 31 December 2011. Cases were allocated into one of five population density groups according to their statistical local area: very low density $(\leqslant 10$ people/km²), low density (11-200 people/km²), medium density (201-1000 people/km²), high density (1001-3000 people/km²), and very high density ( $>3000$ people $/ \mathrm{km}^{2}$ ).

Main outcome measures: Survival to hospital and survival to hospital discharge.

Results: The EMS attended 27705 adult presumed cardiac OHCA cases across 204 Victorian regions. In 12007 of these (43.3\%), resuscitation was attempted by the EMS. Incidence was lower and arrest characteristics were consistently less favourable for lower population density groups. Survival outcomes, including return of spontaneous circulation, survival to hospital and survival to hospital discharge, were significantly poorer in less densely populated groups $(P<0.001$ for all comparisons). When compared with very low density populations, the risk-adjusted odds ratios of surviving to hospital discharge were: low density, 1.88 (95\% Cl, 1.15-3.07); medium density, 2.49 (95\% Cl, 1.55-4.02); high density, 3.47 (95\% Cl, 2.20-5.48) and very high density, 4.32 (95\% Cl, 2.67-6.99).

Conclusion: Population density is independently associated with survival after $\mathrm{OHCA}$, and significant variation in the incidence and characteristics of these events are observed across the state.

study was granted by the Monash University Human Research Ethics Committee.

\section{Setting}

As at 30 June 2007, about 5.2 million people inhabited the state of Victoria, Australia, covering a geographical area of $227000 \mathrm{~km}^{2} .13$ The state comprises 205 inhabited statistical local areas (SLAs) ranging in population density from 0.2 to 7000 people $/ \mathrm{km}^{2}$, and a further four uninhabited SLAs. ${ }^{13}$ The vast majority of its population, about 3.8 million people, reside in the city of Melbourne.

\section{Emergency medical service}

The state operates a single EMS comprising about 2500 paramedics who respond in a two-tier system. Advanced life support (ALS) paramedics are authorised to undertake laryngeal mask airway insertion and administer intravenous adrenaline (epinephrine) during cardiac arrest. Mobile intensive care ambulance (MICA) paramedics provide a higher level of clinical care, performing endotracheal intubation, including rapid sequence intubation, and administer a wider range of cardiotropic medications.

ALS and MICA paramedics are dispatched concurrently to suspected cardiac arrest events in the community. A first responder program for early defibrillation by firefighters and volunteer community teams operates in areas of Melbourne and select rural communities across the state. ${ }^{14}$ For patients with cardiac arrest, paramedic treatment guidelines follow the recommendations of the Australian Resuscitation Council. ${ }^{15}$ Resuscitation efforts can be withheld by paramedics in accordance with clinical practice guidelines, and include circumstances where there is strong evidence of prolonged cardiac arrest, or where patient injuries are incompatible with life.

\section{Data sources}

The VACAR has been described previously. ${ }^{16}$ Electronically recorded patient data are synchronised daily with a central organisational clinical database. Potential cases are identified by VACAR using a highly sensitive search 
1 Patient characteristics and survival outcomes across population densities after adult out-of-hospital cardiac arrest of presumed cardiac aetiology treated by the emergency medical service (EMS) in Victoria, 2003-2011*

\begin{tabular}{|c|c|c|c|c|c|c|c|}
\hline \multirow[b]{2}{*}{ Characteristics and outcomes } & \multirow[b]{2}{*}{ Statewide } & \multicolumn{5}{|c|}{ Population density ${ }^{\dagger}$} & \multirow[b]{2}{*}{$P$} \\
\hline & & Very low & Low & Medium & High & Very high & \\
\hline Total sample & 12007 & 842 & 1709 & 1993 & 6017 & 1446 & \\
\hline Median age in years (IQR) & $70(58-80)$ & $67(56-78)$ & $68(56-77)$ & $68(56-78)$ & $72(60-81)$ & $71(59-80)$ & $<0.001$ \\
\hline Men & $8460(70.5 \%)$ & $626(74.3 \%)$ & $1201(70.3 \%)$ & $1411(70.8 \%)$ & $4148(68.9 \%)$ & $1074(74.3 \%)$ & $<0.001$ \\
\hline Median EMS response time in minutes (IQR) & $8.0(6.0-10.5)$ & $13.0(8.0-21.0)$ & $9.1(7.0-13.0)$ & $9.0(7.0-11.7)$ & $7.5(6.0-9.0)$ & $7.0(5.0-9.0)$ & $<0.001$ \\
\hline Patients with unknown EMS response time & $44(0.4 \%)$ & $11(1.3 \%)$ & $13(0.8 \%)$ & $11(0.6 \%)$ & $8(0.1 \%)$ & $1(0.1 \%)$ & - \\
\hline $\begin{array}{l}\text { Median distance between scene and nearest } \\
\text { EMS station in km (IQR) }\end{array}$ & $2.7(1.6-4.0)$ & $7.3(1.4-18.6)$ & $3.2(1.8-6.7)$ & $3.0(1.8-4.5)$ & $2.7(1.7-3.7)$ & $2.0(1.4-2.6)$ & $<0.001$ \\
\hline \multicolumn{8}{|l|}{ Location of arrest } \\
\hline Home & $8428(70.2 \%)$ & $629(74.7 \%)$ & $1244(72.8 \%)$ & $1435(72.0 \%)$ & $4273(71.0 \%)$ & $847(58.6 \%)$ & $<0.001$ \\
\hline Nursing home & 935 (7.8\%) & $23(2.7 \%)$ & $85(5.0 \%)$ & $135(6.8 \%)$ & $577(9.6 \%)$ & $115(8.0 \%)$ & $<0.001$ \\
\hline Public place & $2557(21.3 \%)$ & $169(20.1 \%)$ & $360(21.1 \%)$ & $408(20.5 \%)$ & $1140(18.9 \%)$ & $480(33.2 \%)$ & $<0.001$ \\
\hline Other & $87(0.7 \%)$ & $21(2.5 \%)$ & $20(1.2 \%)$ & $15(0.8 \%)$ & $27(0.4 \%)$ & $4(0.3 \%)$ & $<0.001$ \\
\hline \multicolumn{8}{|l|}{ First monitored rhythm } \\
\hline VF/VT & $4780(39.8 \%)$ & 299 (35.5\%) & $744(43.5 \%)$ & $830(41.6 \%)$ & $2332(38.8 \%)$ & 575 (39.8\%) & $<0.001$ \\
\hline Asystole & $4567(38.0 \%)$ & $385(45.7 \%)$ & $646(37.8 \%)$ & $742(37.2 \%)$ & 2266 (37.7\%) & $528(36.5 \%)$ & $<0.001$ \\
\hline Pulseless electrical activity & $2476(20.6 \%)$ & $108(12.8 \%)$ & $303(17.7 \%)$ & $402(20.2 \%)$ & $1342(22.3 \%)$ & $321(22.2 \%)$ & $<0.001$ \\
\hline Not shockable & $94(0.8 \%)$ & $13(1.5 \%)$ & $3(0.2 \%)$ & $6(0.3 \%)$ & $55(0.9 \%)$ & $17(1.2 \%)$ & $<0.001$ \\
\hline Unknown - pulseless & $72(0.6 \%)$ & $28(3.3 \%)$ & $10(0.6 \%)$ & $9(0.5 \%)$ & $20(0.3 \%)$ & $5(0.3 \%)$ & $<0.001$ \\
\hline Unknown & $18(0.1 \%)$ & $9(1.1 \%)$ & $3(0.2 \%)$ & $4(0.2 \%)$ & $2(0.0 \%)$ & 0 & - \\
\hline \multicolumn{8}{|l|}{ Highest skill level on scene } \\
\hline Basic life support & $643(5.4 \%)$ & $174(20.7 \%)$ & $116(6.8 \%)$ & $105(5.3 \%)$ & $199(3.3 \%)$ & $49(3.4 \%)$ & $<0.001$ \\
\hline Advanced life support & $907(7.6 \%)$ & $200(23.8 \%)$ & $113(6.6 \%)$ & $141(7.1 \%)$ & $368(6.1 \%)$ & $85(5.9 \%)$ & $<0.001$ \\
\hline Intensive care & 9091 (75.7\%) & 361 (42.9\%) & $1238(72.4 \%)$ & $1498(75.2 \%)$ & $4818(80.1 \%)$ & $1176(81.3 \%)$ & $<0.001$ \\
\hline Unknown & $1366(11.4 \%)$ & $107(12.7 \%)$ & $242(14.2 \%)$ & $249(12.5 \%)$ & $632(10.5 \%)$ & $136(9.4 \%)$ & $<0.001$ \\
\hline Bystander witnessed & 7316 (60.9\%) & $519(61.6 \%)$ & $1070(62.6 \%)$ & $1267(63.6 \%)$ & $3561(59.2 \%)$ & $899(62.2 \%)$ & $<0.001$ \\
\hline Unknown & 69 (0.6\%) & $12(1.4 \%)$ & $15(0.9 \%)$ & $12(0.6 \%)$ & $24(0.4 \%)$ & $6(0.4 \%)$ & - \\
\hline Bystander CPR performed & $5803(48.3 \%)$ & $497(59.0 \%)$ & $924(54.1 \%)$ & 1029 (51.6\%) & 2699 (44.9\%) & $654(45.2 \%)$ & $<0.001$ \\
\hline Unknown & $580(4.8 \%)$ & $36(4.3 \%)$ & $75(4.4 \%)$ & $90(4.5 \%)$ & $284(4.7 \%)$ & $95(6.6 \%)$ & - \\
\hline \multicolumn{8}{|l|}{ Scene outcomes } \\
\hline Efforts ceased at scene & $7397(61.6 \%)$ & $632(75.1 \%)$ & $1089(63.7 \%)$ & $1250(62.7 \%)$ & $3615(60.1 \%)$ & 811 (56.1\%) & $<0.001$ \\
\hline Transport with ROSC & $3625(30.2 \%)$ & $104(12.4 \%)$ & $401(23.5 \%)$ & $554(27.8 \%)$ & $2046(34.0 \%)$ & $52(36.0 \%)$ & $<0.001$ \\
\hline Transport with CPR ongoing & $985(8.2 \%)$ & $106(12.6 \%)$ & $219(12.8 \%)$ & $189(9.5 \%)$ & $356(5.9 \%)$ & $115(8.0 \%)$ & $<0.001$ \\
\hline ROSC at any time & $4272(35.6 \%)$ & $128(15.2 \%)$ & $469(27.4 \%)$ & $659(33.1 \%)$ & $2430(40.4 \%)$ & $586(40.5 \%)$ & $<0.001$ \\
\hline Survival to hospital ${ }^{\ddagger}$ & 3655 (30.7\%) & $101(12.3 \%)$ & $408(24.2 \%)$ & $559(28.3 \%)$ & $2058(34.4 \%)$ & $529(36.9 \%)$ & $<0.001$ \\
\hline Unknown & $102(0.8 \%)$ & $20(2.4 \%)$ & $20(1.2 \%)$ & $17(0.9 \%)$ & $34(0.6 \%)$ & $11(0.8 \%)$ & - \\
\hline Survival to hospital discharge ${ }^{\ddagger}$ & $1176(10.0 \%)$ & $23(2.8 \%)$ & $117(7.1 \%)$ & $171(8.8 \%)$ & $649(10.9 \%)$ & $216(15.2 \%)$ & $<0.001$ \\
\hline Unknown & $218(1.8 \%)$ & $27(3.2 \%)$ & $56(3.3 \%)$ & $41(2.1 \%)$ & $72(1.2 \%)$ & $22(1.5 \%)$ & - \\
\hline
\end{tabular}

* Values are numbers (\%) of patients unless otherwise indicated. † Population density categories were defined as: very low density, $\leqslant 10$ people/km²; low density, $11-200$ people/ $/ \mathrm{km}^{2}$; medium density, 201-1000 people/ $\mathrm{km}^{2}$; high density, 1001-3000 people/km²; and very high density, >3000 people/km². ‡Survival figures exclude missing data. $\mathrm{CPR}=$ cardiopulmonary resuscitation. IQR = interquartile range. $\mathrm{ROSC}=$ return of spontaneous circulation. VF/VT = ventricular fibrillation or pulseless ventricular tachycardia.

strategy, and individually screened for eligibility. All eligible cases are reviewed and entered into the registry according to the Utstein requirements. ${ }^{17}$ Hospital follow-up data are obtained from hospital medical records in as many as $99 \%$ of all transported cases.

\section{Definitions}

Cardiac arrests are presumed to be of cardiac aetiology unless otherwise stated on the patient care record. The variable "EMS response time" is defined as the time from emergency call to arrival of the first emergency medical response team to the scene. Bystander cardiopulmonary resuscitation (CPR) is defined as any attempt at chest compressions, with or without ventilations. Survival to hospital is defined as a palpable carotid pulse on arrival at hospital as documented on the patient care record.

\section{Geospatial and statistical analyses}

Spatial data of included cases were defined by region according to 209
SLAs. Geospatial mapping software MapInfo Professional version 11.5 (Pitney Bowes Software) was used to assign population characteristics according to the longitude and latitude coordinates of the case scene location. Scene address was used to generate approximate location coordinates in cases with missing data. Age-stratified population characteristics for OHCA cases were obtained from the Australian Bureau of Statistics for population estimates at 30 June 2007.13 
2 Crude and adjusted odds ratios (and $95 \% \mathrm{Cl}$ ) for effect of population density on risk-adjusted odds of survival $(95 \% \mathrm{Cl})$ in population treated by the emergency medical service (EMS) and in ventricular fibrillation or pulseless ventricular tachycardia (VF/VT) subgroup, after adult out-ofhospital cardiac arrest of presumed cardiac aetiology in Victoria, 2003-2011*

EMS-treated population

VF/VT subgroup

\begin{tabular}{|c|c|c|c|c|c|c|c|c|}
\hline \multirow[b]{2}{*}{$\begin{array}{l}\text { Population } \\
\text { density (people/ } \\
\mathrm{km}^{2} \text { ) }\end{array}$} & & \\
\hline & $\begin{array}{l}\text { Crude survival } \\
\text { to hospital }\end{array}$ & $\begin{array}{l}\text { Adjusted } \\
\text { survival to } \\
\text { hospital }\end{array}$ & $\begin{array}{l}\text { Crude survival } \\
\text { to hospital } \\
\text { discharge }\end{array}$ & $\begin{array}{l}\text { Adjusted } \\
\text { survival to } \\
\text { hospital } \\
\text { discharge }\end{array}$ & $\begin{array}{l}\text { Crude survival } \\
\text { to hospital }\end{array}$ & $\begin{array}{l}\text { Adjusted } \\
\text { survival to } \\
\text { hospital }\end{array}$ & $\begin{array}{l}\text { Crude survival } \\
\text { to hospital } \\
\text { discharge }\end{array}$ & $\begin{array}{l}\text { Adjusted } \\
\text { survival to } \\
\text { hospital } \\
\text { discharge }\end{array}$ \\
\hline Very low $(\leqslant 10)$ & $1.00^{\dagger}$ & & & & $1.00^{\dagger}$ & & & \\
\hline $\begin{array}{l}\text { Low } \\
(11-200)\end{array}$ & $\begin{array}{c}2.27 \\
(1.80-2.88)^{\ddagger}\end{array}$ & $\begin{array}{c}2.19 \\
(1.69-2.84)^{\ddagger}\end{array}$ & $\begin{array}{c}2.62 \\
(1.66-4.14)^{\ddagger}\end{array}$ & $\begin{array}{c}1.88 \\
(1.15-3.07)^{\S}\end{array}$ & $\begin{array}{c}1.78 \\
(1.30-2.45)^{\ddagger}\end{array}$ & $\begin{array}{c}1.87 \\
(1.34-2.60)^{\ddagger}\end{array}$ & $\begin{array}{c}1.93 \\
(1.19-3.13)^{\S}\end{array}$ & $\begin{array}{c}1.60 \\
(0.96-2.65)\end{array}$ \\
\hline $\begin{array}{l}\text { Medium } \\
(201-1000)\end{array}$ & $\begin{array}{c}2.82 \\
(2.24-3.54) \ddagger\end{array}$ & $\begin{array}{c}2.86 \\
(2.21-3.69) \ddagger\end{array}$ & $\begin{array}{c}3.31 \\
(2.12-5.20) \ddagger\end{array}$ & $\begin{array}{c}2.49 \\
(1.55-4.02)^{\ddagger}\end{array}$ & $\begin{array}{c}2.48 \\
(1.82-3.39) \ddagger\end{array}$ & $\begin{array}{c}2.53 \\
(1.83-3.50)^{\ddagger}\end{array}$ & $\begin{array}{c}2.63 \\
(1.64-4.21) \ddagger\end{array}$ & $\begin{array}{c}2.12 \\
(1.30-3.45)^{\S}\end{array}$ \\
\hline $\begin{array}{l}\text { High } \\
\text { (1001-3000) }\end{array}$ & $\begin{array}{c}3.74 \\
(3.02-4.64)^{\ddagger}\end{array}$ & $\begin{array}{c}4.15 \\
(3.26-5.29)^{\ddagger}\end{array}$ & $\begin{array}{c}4.22 \\
(2.77-6.44)^{\ddagger}\end{array}$ & $\begin{array}{c}3.47 \\
(2.20-5.48)^{\ddagger}\end{array}$ & $\begin{array}{c}3.92 \\
(2.94-5.24)^{\ddagger}\end{array}$ & $\begin{array}{c}4.10 \\
(3.01-5.56)^{\ddagger}\end{array}$ & $\begin{array}{c}3.73 \\
(2.39-5.82)^{\ddagger}\end{array}$ & $\begin{array}{c}2.96 \\
(1.86-4.72)^{\ddagger}\end{array}$ \\
\hline Very high (> 3000) & $\begin{array}{c}4.17 \\
(3.30-5.27)^{\ddagger}\end{array}$ & $\begin{array}{c}4.37 \\
(3.35-5.69)^{\ddagger}\end{array}$ & $\begin{array}{c}6.16 \\
(3.97-9.55)^{\ddagger}\end{array}$ & $\begin{array}{c}4.32 \\
(2.67-6.99)^{\ddagger}\end{array}$ & $\begin{array}{c}4.53 \\
(3.28-6.26)^{\ddagger}\end{array}$ & $\begin{array}{c}4.42 \\
(3.14-6.23)^{\ddagger}\end{array}$ & $\begin{array}{c}5.94 \\
(3.72-9.50)^{\ddagger}\end{array}$ & $\begin{array}{c}3.87 \\
(2.36-6.35)^{\ddagger}\end{array}$ \\
\hline
\end{tabular}

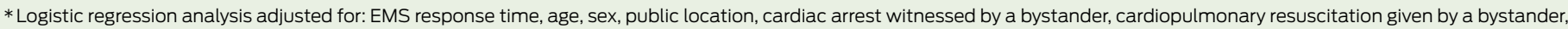
and VF/VT (EMS-treated patients only). † Reference category. $\ddagger P<0.001$. $\$ P<0.05$.
For the analysis, cases were stratified into five population density categories according to the population density of their immediate SLA: very low density $\left(\leqslant 10\right.$ people $\left./ \mathrm{km}^{2}\right)$, low density (11-200 people $\left./ \mathrm{km}^{2}\right)$, medium density (201-1000 people/ $\mathrm{km}^{2}$ ), high density (1001-3000 people $\left./ \mathrm{km}^{2}\right)$, and very high density ( $>3000$ people $/ \mathrm{km}^{2}$ ). Patient characteristics and survival outcomes were compared across denthe Kruskal-Wallis test as appropriate. The Victorian standard population as at 30 June 2007 was used to calculate the adjusted incidences in each region.

The effect of population density on survival outcome was assessed using a logistic regression model in the overall EMS-treated population and the ventricular fibrillation or pulseless ventricular tachycardia (VF/VT) subgroup. Multivariate models were adjusted for factors with known associations to survival, including: EMS response time, age, sex, public location, the event being witnessed by a bystander, bystander CPR and VF/ VT (EMS-treated population only). Independent variables were chosen on the basis of previous reports, ${ }^{16,18}$ and underwent testing in our models to evaluate potential interaction effects with survival (eg, EMS response time and population density). The very low density group was expressed as the reference category in all models. Effect sizes were presented as risk-adjusted odds ratios (AOR) and 95\% CIs. P less significant. The predicted probability of survival was graphically depicted after conditional standardisation. sity groups using the Pearson $\chi^{2}$ test or than 0.05 was considered statistically
Statistical analyses were undertaken using PASW Statistics 18 (SPSS).

\section{Results}

The EMS attended 27705 adult presumed cardiac OHCA cases across 204 Victorian SLAs (Appendix 1; online at mja.com.au). In 12007 of these (43.3\%), the EMS attempted resuscitation (the EMS-treated sample).

\section{Sample characteristics and incidence rates}

The statewide unadjusted incidence in the EMS-attended sample was 76.7 per 100000 adult person-years and in the EMS-treated sample it was 33.3 per 100000 adult person-years. The age-adjusted incidence rate, mortality rate and case fatality rate also varied across population densities (Appendix 2; online at mja.com.au).

A lower median age was observed in the lower population density groups when compared with higher density groups $(P<0.001$, Box 1$)$. EMS response time and the median distance of the nearest EMS station from the scene were significantly higher in the lower density populations. Arrests in public locations and aged care facilities were more frequent in higher density groups. With the exception of the very low density group, VF/VT accounted for about $40.0 \%$ of the first monitored rhythms. While the proportion of bystander-witnessed events was similar between the very high and very low density groups (62.2\% v $61.6 \%, P=0.962)$, the proportion of patients receiving bystander CPR
3 Predicted probability of survival* $(95 \% \mathrm{Cl})$ in adult out-of-hospital cardiac arrest of presumed cardiac aetiology treated by the emergency medical service in Victoria, 2003-2011

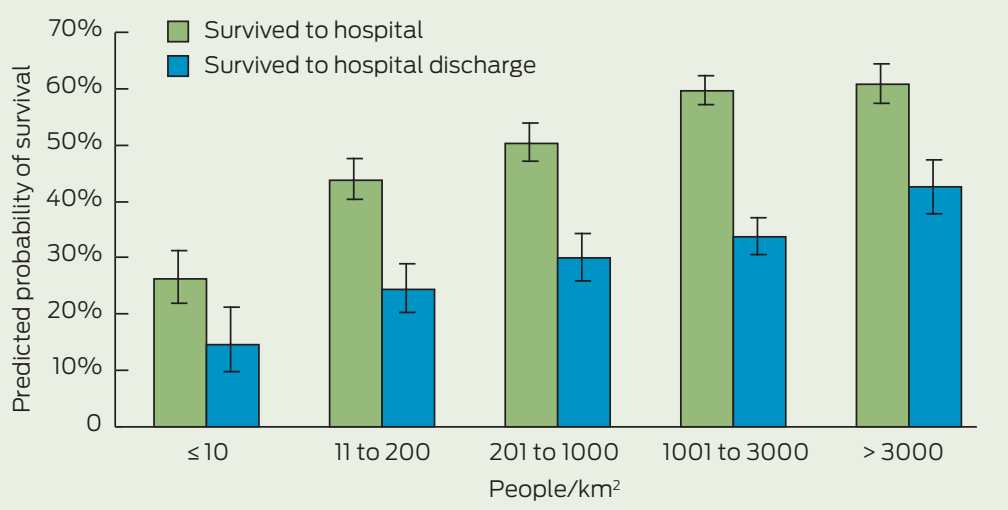

* Conditional probability of survival calculated for a 60 -year-old man who suffers a bystanderwitnessed ventricular fibrillation or pulseless ventricular tachycardia cardiac arrest in a public place and receives cardiopulmonary resuscitation from a bystander. Emergency medical service response time is 9 minutes. 
4 Annual unadjusted case fatality rates across population densities in adult out-of-hospital cardiac arrest of presumed cardiac aetiology treated by the emergency medical service in Victoria

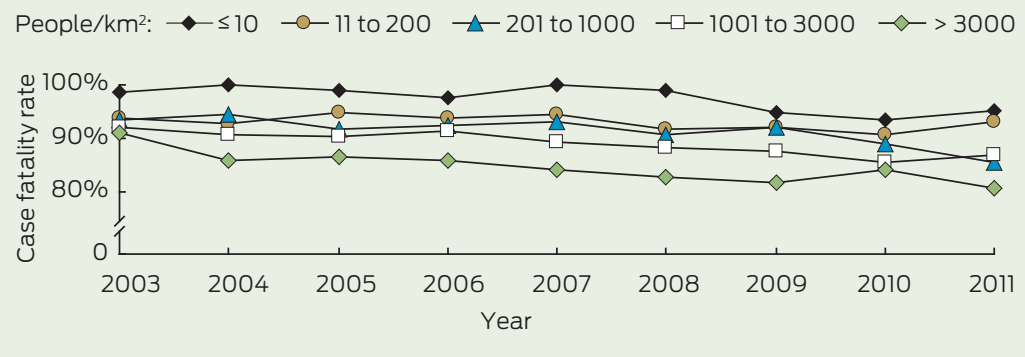

varied significantly (45.2\% v 59.0\%, $P<0.001)$.

\section{Outcomes}

Unadjusted survival outcomes were consistently in favour of high density groups (Box 1). Univariate comparisons of return of spontaneous circulation $(40.5 \%$ v $15.2 \%, P<0.001)$, survival to hospital $(36.9 \% \mathrm{v} 12.3 \%, P<0.001)$, and survival to hospital discharge $(15.2 \%$ v $2.8 \%, P<0.001)$ were significantly improved in the very high density group when compared with the very low density group. After adjusting for known survival confounders, the AOR of survival to hospital and survival to hospital discharge were strongly associated with density group across both the overall EMS-treated population and the VF/VT subgroup (Box 2). The predicted probability of survival after standardisation (Box 3 ) and the annual unadjusted case fatality rates (Box 4) were improved with higher density groups.

\section{Discussion}

This study identifies that population density is independently associated with survival outcome from OHCA, with trends observed in its incidence and characteristics. The data collected for this study are representative of $99.5 \%$ of the inhabited regions of Victoria, and offer a key strength over previous reports, which may have been limited to data collected from select municipalities. ${ }^{6-8}$

Variation in the incidence of $\mathrm{OHCA}$ has been described previously, ${ }^{1,8}$ with the global variation of EMS-attended OHCA ranging between 24.0 and 186.0 per 100000 person-years. ${ }^{1}$ In the current study, the highest incidence of OHCA was found in the very high density region, where the presence of modifiable cardiovascular risk factors is reportedly lower than the Australian average. ${ }^{19}$ This paradox can be partly explained by a shift in Melbourne's diurnal population where people commuting to areas of work create a sixfold increase in the normal resident population of inner Melbourne. ${ }^{20}$ This may also explain the increased public nature of arrests in the very high density group. Despite significantly better outcomes in higher density areas, lower density groups were associated with favourable baseline characteristics such as younger age, greater bystander CPR and increased VF/VT (low density group). While these findings are likely to be interdependent, they may also indicate selectivity in the EMS treated population, where poorer response time performance is often mitigated by the presence of younger patients and by bystander-initiated resuscitation. ${ }^{21}$

While previous authors have coupled poorer survival in regional areas to increased EMS response times, this study shows that the association between population density and survival is independent of known survival confounders. Authors from Japan have described similar findings, ${ }^{10}$ and suggest that combinations of prehospital and hospital-based factors are contributing to regional disparities in OHCA outcome. For example, our study showed a disparity in the availability of intensive care paramedics in less densely populated areas of Victoria. While the impact of paramedic skill level on OHCA outcome is yet to be well understood, an Australian report conducted in a similar EMS system showed that access to an intensive care paramedic was associated with a significant increase in survival to hospital discharge after OHCA. ${ }^{22}$ Reduced paramedic exposure to resuscitation practice in lower density areas is also likely to be a contributing factor, although evidence in this area remains scarce and requires further exploration. In addition, the frequency of preventable bystander delays after OHCA has undergone a recent evaluation, which shows that arrests occurring in rural regions were independently associated with emergency call delays and death after OHCA.21

Similarly, hospital-based factors have been associated with regional variation in $\mathrm{OHCA}$ outcomes. A recent report from Victoria established that the availability of a 24-hour cardiac interventional facility could partly explain the variation in regional survival from OHCA. ${ }^{23}$ The greatest survival benefit was observed in patients transported to two centralised trauma hospitals situated in the very high density population of Melbourne, and may partly explain the increased survival observed in this region. While the importance of regionalised trauma centres has been well established in Victoria, the implementation of centralised care pathways for OHCA is less certain. ${ }^{24}$ In fact, few randomised controlled trials have been conducted to examine the benefit of system pathways that improve access to sophisticated emergency teams and optimal post-arrest practices.2,24 These clinical trials are urgently needed, and will help inform the contribution of regionalised post-arrest care on OHCA outcomes in Australia.

We acknowledge several limitations in this study. First, its retrospective design carries inherent limitations. Higher levels of missing survival data were present in the lower density groups and may have introduced some bias. Second, our population density categories were arbitrarily chosen and may not be generalisable in cross-nation comparisons. Finally, other confounding factors such as transport time to hospital and paramedic skill level were considered, but not included in our multivariate analyses. Cases not involving intensive care paramedics were presumed to be overrepresented by futile attempts at resuscitation, and excluding them reduces the possibility of confounding in our models. Similarly, while transport time offers an appropriate surrogate of time to definitive care, its inclusion in multivariate models 
provides little prognostic information, ${ }^{25}$ and excludes patients whose resuscitation efforts are ceased at scene (greater in lower density areas).

Nevertheless, this study provides key insight into the variation in OHCA outcomes observed across a large Australian community, and indicates that population density is independently associated with survival outcome. However, the causative factors associated with the observed variation in outcomes remain speculative and require further investigation. Delineating prehospital and inhospital factors associated with improved outcomes in densely populated areas can be used to inform practice recommendations and service provision in sparse populations.

Acknowledgements: Janet Bray receives salary support from the National Health and Medical Research Council (NHMRC) Australian Resuscitation Outcomes Consortium (Aus-ROC) Centre of Research Excellence 1029983 (https://www.ausroc.org.au) and a co-funded NHMRC/ National Heart Foundation Fellowship (1069985).

\section{Competing interests: No relevant disclosures.}

Received 25 Jun 2013, accepted 26 Nov 2013.

1 Berdowski J, Berg RA, Tijssen JG, Koster RW. Global incidences of out-of-hospital cardiac arrest and survival rates: systematic review of 67 prospective studies. Resuscitation 2010; 81 : 1479-1487.

2 Nichol G, Aufderheide TP, Eigel B, et al. Regional systems of care for out-of-hospital cardiac arrest: a policy statement from the American Heart Association. Circulation 2010; 121 : 709-729.

3 Heeley EL, Wei JW, Carter K, et al. Socioeconomic disparities in stroke rates and outcome: pooled analysis of stroke incidence studies in Australia and New Zealand. Med J Aust 2011; 195: 10-14.

4 Ranasinghe I, Turnbull F, Tonkin A, et al. Comparative effectiveness of population interventions to improve access to reperfusion for ST-segment-elevation myocardial infarction in Australia. Circ Cardiovasc Qual Outcomes 2012; 5: 429-436.
5 Fatovich DM, Phillips M, Langford SA, Jacobs IG. A comparison of metropolitan vs rural major trauma in Western Australia. Resuscitation 2011; 82: 886-890.

6 Hiltunen P, Kuisma M, Silfvast T, et al. Regional variation and outcome of out-of-hospital cardiac arrest (ohca) in Finland - the Finnresusci study. Scand J Trauma Resusc Emerg Med 2012; 20: 80 .

7 Strömsöe A, Svensson L, Claesson A, et al. Association between population density and reported incidence, characteristics and outcome after out-of-hospital cardiac arrest in Sweden. Resuscitation 2011; 82: 1307-1313.

8 Nichol G, Thomas E, Callaway CW, et al. Regional variation in out-of-hospital cardiac arrest incidence and outcome. JAMA 2008; 300: 1423-1431.

9 Wang HE, Devlin SM, Sears GK, et al. Regional variations in early and late survival after outof-hospital cardiac arrest. Resuscitation 2012; 83: 1343-1348.

10 Yasunaga $\mathrm{H}$, Miyata $\mathrm{H}$, Horiguchi $\mathrm{H}$, et al. Population density, call-response interval, and survival of out-of-hospital cardiac arrest. Int J Health Geogr 2011; 10: 26

11 Jennings PA, Cameron P, Walker T, et al. Outof-hospital cardiac arrest in Victoria: rural and urban outcomes. Med J Aust 2006; 185: 135-139.

12 Young B, Woodall J, Enraght-Moony E, et al. Rural and remote cardiac outcomes: examination of a state-wide emergency medical service. Proceedings of the 9 th National Rural Health Conference; 2007 Mar 7-10; Albury, New South Wales. http:// ruralhealth.org.au/9thNRHC/9thnrhc. ruralhealth.org.au/program/docs/papers/ young_C3.pdf (accessed Dec 2013).

13 Australian Bureau of Statistics. Regional population growth, Australia, 2006-2007. Canberra: ABS, 2008. (ABS Cat. No. 3218.0.) http://www.abs.gov.au/AUSSTATS/abs@.nsf/ DetailsPage/3218.02006-07?OpenDocument (accessed Mar 2013).

14 Smith KL, McNeil JJ; Emergency Medical Response Steering Committee. Cardiac arrests treated by ambulance paramedics and fire fighters. Med J Aust 2002; 177: 305-309.

15 Australian Resuscitation Council, New Zealand Resuscitation Council. Protocols for adult advanced life support. ARC and NZRC Guideline 2010. Emerg Med Australas 2011; 23: 271-274.

16 Fridman M, Barnes V, Whyman A, et al. A model of survival following pre-hospital cardiac arrest based on the Victorian Ambulance Cardiac Arrest Register. Resuscitation 2007; 75: 311-322.
17 Jacobs I, Nadkarni V, Bahr J, et al. Cardiac arrest and cardiopulmonary resuscitation outcome reports: update and simplification of the Utstein templates for resuscitation registries. A statement for healthcare professionals from a task force of the international liaison committee on resuscitation (American Heart Association, European Resuscitation Council, Australian Resuscitation Council, New Zealand Resuscitation Council, Heart and Stroke Foundation of Canada, InterAmerican Heart Foundation, Resuscitation Council of Southern Africa). Resuscitation 2004; 63: 233-249.

18 Sasson C, Rogers MA, Dahl J, Kellermann AL. Predictors of survival from out-of-hospital cardiac arrest: a systematic review and metaanalysis. Circ Cardiovasc Qual Outcomes 2010; 3: 63-81.

19 Public Health Information Development Unit. Population health profile of the Melbourne Division of General Practice. Population Profile Series: No. 38. Adelaide: PHIDU, 2005. http://www.publichealth.gov.au/ pdf/profiles/2005/301_Melbourne_DGP.pdf (accessed Jun 2013).

20 Department of Planning and Community Development. Demographic characteristics of communities within the Melbourne Investigation Area. Melbourne: DPCD, Victorian Government, 2009. http://www.veac.vic.gov. au/documents/VEAC\%20demographics $\% 20$ report\%20Final\%2014.7.09.pdf (accessed Jun 2013).

21 Nehme Z, Andrew E, Cameron P, et al. Direction of first bystander call for help is associated with outcome from out-of-hospital cardiac arrest. Resuscitation 2013; 4 Sep [Epub ahead of print] doi: 10.1016/j.resuscitation.2013.08.258.

22 Woodall J, McCarthy M, Johnston T, et al. Impact of advanced cardiac life support-skilled paramedics on survival from out-of-hospital cardiac arrest in a statewide emergency medical service. Emerg Med J 2007; 24: 134-138.

23 Stub D, Smith K, Bray JE, et al. Hospital characteristics are associated with patient outcomes following out-of-hospital cardiac arrest. Heart 2011; 97: 1489-1494.

24 Stub D, Bernard S, Smith K, et al. Do we need cardiac arrest centres in Australia? Intern Med J 2012; 42: 1173-1179.

25 Cudnik MT, Schmicker RH, Vaillancourt C et al. A geospatial assessment of transport distance and survival to discharge in out of hospital cardiac arrest patients: implications for resuscitation centers. Resuscitation 2010; 81: 518-523. 